\title{
Cognitive simulation modeling on the VAX-11
}

\author{
ALAN M. LESGOLD \\ Learning Research and Development Center, University of Pittsburgh, Pittsburgh, Pennsylvania 15260
}

The ability of the VAX-11 to serve as both a real-time and a large-memory-space machine is described. The issue of how to do cognitive simulation work on the VAX-11 is addressed.

\begin{abstract}
A large minicomputer like the VAX-11 is a tempting item for any laboratory that can afford one, because of its ability to be both a real-time machine and a largememory-space machine. In this report, I concentrate on the latter capability and address the issue of how to do cognitive simulation work on the VAX-11. As this is a relatively new architecture, there is not yet the same cornucopia of existing software for artificial intelligence efforts as is found on such systems as the DECsystems 10 and 20 . Consequently, a bit more preparation is required before one can get into the cognitive science business on a VAX-11. I will address three issues of concern: memory capacity, disk space, and language and programmer environment support.
\end{abstract}

\section{MEMORY}

The VAX-11 is a true virtual memory machine. As such, the size of program that can be executed on it is not restricted by the amount of physical memory that it contains. Nonetheless, memory is a significant factor in system performance. In our own laboratory, we have seen a number of occasions in which several massive-sized programs running simultaneously require so much paging (switching of pieces of the program space from disk to memory and back) that the processor itself sits idle for perhaps one-third of the available cycles. Also, having inadequate memory may increase bus traffic in ways that interfere with real-time inputs that may be required by other users. If cognitive simulators and experimentalists are to coexist on the same machine, it must be configured with an appropriate assignment of devices to MASSBUS and UNIBUS channels and with enough physical memory to avoid intolerable paging problems.

However, memory is becoming less expensive, and it is sold in small units (about $\$ 1,400$ for 256 Kbytes from second sources, as of this writing). Consequently, it may be worthwhile to settle other problems, such as

VAX-11, UNIBUS, MASSBUS, and VAX/VMS are trademarks of Digital Equipment Corporation, UNIX is a trademark of Western Electric, and EUNICE is a trademark of SRI International. The information presented here is of a sort that is subject to change and is based upon inferences that go some distance beyond the available data. It should be used with caution. disk space, first, since those problems require more study and larger expenditures.

\section{DISK SPACE}

Large-scale programs and data bases take up considerable storage space. In our installation, the largest projects require about 20 megabytes of on-line storage and would probably operate more efficiently with twice that space. In addition, word-processing activity is easily fit into the spare capacity of the machine and is a lowsystem-demand activity, so it is tempting to use the VAX-11 as a means of modernizing office functions. However, a productive researcher may have hundreds of pages of manuscripts in progress at any given instant, may want old papers on-line to borrow from, and so on. Thus, word processing can significantly increase disk usage. The moral is this: Get all the disk space you can.

There are basically three ways to expand the disk space in a VAX-11 system. To provide a sense of pricing differences in these options, I will describe a standard arrangement to provide 300 megabytes of unformatted disk space as an add-on to an existing system. The first possibility is to buy the added disk support from Digital Equipment Corporation (DEC). Their Model RM-05 provides the 300 megabytes (unformatted) and currently costs $\$ 34,000$ as an add-on to an existing MASSBUS handling RM-type drives. (They also offer a fixedmedium, Winchester-type, drive that contains 154 megabytes for $\$ 20,000$.) Buying the add-on equipment from the manufacturer simplifies life considerably. Software support is guaranteed (if the operating system used is the standard manufacturer product, VAX/VMS), maintenance is straightforward (DEC will maintain the drive with the rest of the system), and reliability is likely to be the highest possible. On the other hand, the equipment is quite expensive.

The second and third alternatives involve buying equipment from other manufacturers. There are a number of manufacturers of 300-megabyte storage module drives and a number of manufacturers that make disk controllers for the VAX-11. Their products are often sold at significantly lower prices than DEC products with the same storage capacity. All of these alternative controllers have potential weak points that need to be 
examined: (1) The companies making the controllers are not all as stable and established as DEC; (2) some of the controllers require modifications (patches) to the driver software provided by DEC in the operating system; (3) patent infringement suits that have yet to be resolved may limit the future manufacture of some of these devices or may force smaller companies out of business; and (4) maintenance contracts may need to be negotiated separately for the drive and the controller, which DEC currently will not maintain. However, the devices are cheaper.

The second alternative is to purchase a controller that either allows direct interfacing to the backplane of the VAX-11 (the SBI) or that is interposed between an existing MASSBUS adaptor and an existing DEC drive. Such possibilities (from Systems Industries and Plessey, and perhaps others) provide the greatest guarantee that disk traffic will not interfere with real-time data traffic on the UNIBUS. However, they involve newer and more complex technology and are more likely to be successful infringement suit targets. Also, they cost $\$ 25,000$ to $\$ 34,000$ for the first add-on drive. Finally, it is not yet established that a MASSBUS can handle highvolume traffic to many drives without degradation of service.

The final alternative is a controller that interfaces to the UNIBUS. These are manufactured by Emulex, Minicomputer Technology, Systems Industries, Advanced Electronic Devices, and other companies. The technology is not that new, as these companies have been producing controllers for the DEC PDP-11 for some time. The price of a controller and a 300-megabyte drive ranges from $\$ 17,000$ to $\$ 25,000$, perhaps slightly lower if you know where to look. The problems that can arise, in addition to the general problems with second-source equipment, have to do with the mix of devices on the UNIBUS. While direct memory channels like UNIBUS have a relatively high throughput rate when measured per character, there is an additional cost per transaction. Some of the controllers may not be optimal in the amount of data transferred in each burst. If bursts are too short, the overhead cost per transaction may clog the UNIBUS. If they are too long, high-priority devices may not be able to gain control of the bus fast enough for time-critical activities.

Regrettably, there is little in the way of guidelines for deciding whether a UNIBUS can handle a large disk drive in addition to everything else. A rule of thumb is that if there is a lot of real-time activity on your UNIBUS, be wary of adding a lot of additional disk storage. Also, a second UNIBUS adaptor is expensive. Consequently, be sure that the UNIBUS option is compatible with long-term plans for UNIBUS traffic, whether due to realtime $\mathrm{I} / \mathrm{O}$ devices or to further disk expansion. One final warning: it has been proposed to some VAX-11 users that they finance the acquisition of second-source disk support by selling their original equipment drives, which may command a much higher price used than secondsource equipment costs new. It is not wise to have the paging and swapping functions carried out via a single UNIBUS. The system disk, which contains the paging and swapping files, should be on the MASSBUS. This probably means that installations should keep at least one original equipment DEC drive.

In summary, you get what you pay for, more or less, in the purchase of disk drives. At least, that is the most you can hope for. Nonetheless, successful uses of secondsource arrangements, as supplements to a DEC drive acting as the system drive, are increasingly common.

\section{LANGUAGES}

Appropriate languages for cognitive simulation work are just beginning to appear for the VAX-11. In this section, I will discuss several alternatives worthy of consideration.

There are three serious contenders for the title of decent LISP system. One of these, Berkeley LISP, depends on the UNIX operating system, and there is some possibility that a second alternative, the ISI Interlisp system, may depend upon UNIX. Thus, the prior question would seem to be, "Which operating system do I want?"

Fortunately, it now appears that this problem can be solved. David Kashtan at SRI International has written a system called EUNICE that allows programs written in $C$ for the UNIX operating system to be executed under VAX/VMS. Berkeley LISP, for example, is written in $\mathrm{C}$, so it can be used either under UNIX directly or under VAX/VMS with the EUNICE package. The EUNICE package has, in fact, been used with Berkeley LISP at the University of California at Berkeley in successful test trials. Thus, we can consider LISP systems independent of operating systems, assuming that the licensing details for EUNICE and UNIX are not a problem. Academic-use licenses for UNIX and for EUNICE cost $\$ 200$ to $\$ 300$ each.

The three top contenders as serious VAX LISP systems are Berkeley LISP (also called FRANZ LISP), Interlisp, and NIL. The first, Berkeley LISP, has the advantage of being in production use today. Interlisp and NIL should both become available over the next year or so. Of the three, I have established that Berkeley LISP and ISI Interlisp will be licensed to academic users at little or no cost. I am not sure about NIL. NIL is an effort by the M.I.T. Artificial Intelligence Laboratory to create a new generation of LISP patterned after MACLISP but taking account of the high-quality programmer environment of Interlisp. Interlisp, originally a product of Bolt, Beranek, and Newman and the Xerox Corporation, is being adapted by the Information Sciences Institute at the University of Southern California for the VAX-11. Berkeley LISP is an existing language that has evolved to the VAX-11 already; it is a 
product of the University of California at Berkeley's Department of Electrical Engineering and Computer Science.

Three other possibilities are also available. At the University of Pittsburgh Learning Research and Development Center, we have been using the LISPF3 subset of Interlisp, coded in FORTRAN. This has allowed a year's head start on our VAX-11, with VAX/VMS. The LISPF3 is a perfectly serviceable language for projects of small to moderate size. It was produced at the University of Uppsala in Sweden. However, it is slow and somewhat inefficient. We are about to replace it with EUNICE and Berkeley LISP. The Department of Computer Science at University of Massachusetts is using a version of DELPHI LISP under VAX/VMS. From examination of the manual, it also looks reasonable. Finally, there is a project in the University of Wisconsin Department of Computer Science to code the basics of Interlisp in PASCAL. The system that they have created is usable under VAX/VMS. However, Interlisp is generally on the slow side, and VAX PASCAL is much slower than VAX FORTRAN, so it is not clear that this possibility, which I have not investigated, will work out well. My personal view is that Interlisp, NIL, and Berkeley LISP all merit careful consideration. Existing programs running on the PDP-10 or some other machine may force any given user to one of these three alternatives. If not, all should be considered. We at the University of Pittsburgh Learning Research and Development Center hope to support both Berkeley LISP and Interlisp, if possible.

\section{THE FUTURE}

Clearly, psychologists will someday want to have intelligent programs, which embody their theories, running in real-time and interacting with subjects in the laboratory. For those of us on university-level research budgets, this will not likely be possible if the approach is to have a knowledge-intensive LISP program doing a real-time experiment; the models being created today run far too slowly. Consequently, we must begin to address the issue of how to spawn subsets of a theoret ical simulation that are efficient enough to interact with subjects in the laboratory. Whether this can be done in the context of existing LISP systems remains to be seen. 